\title{
EN TORNO A LA ACADEMIA DEL \\ ARCADIA: ILUSTRES ADMITIDAS E \\ ILUSTRES EXCLUIDAS
}

\section{AROUND THE ACADEMY OF ARCADIA: ILLUSTRIUS ADMITTED AND ILLUSTRIOUS EXCLUDED}

\section{Juan Aguilar González}

Universidad de Castilla - La Mancha

juan.aguilarg@gmail.com

Recibido: septiembre 2021

Aceptado: noviembre 2021

Palabras clave: Literatura, género, academias, Arcadia.

Keywords: Literature, genre, academies, Arcadia.

Resumen: La Academia de la Arcadia supuso, desde su creación, un excelente auspicio para las mujeres que deseaban ejercer la escritura. Las escritoras de la Arcadia formaron un nutrido grupo que, si bien dentro del canon impuesto, destacó por la calidad de algunas de sus componentes hasta el punto de llegar a formar parte de las antologías masculinas de la época y siglos posteriores.

No obstante, no todo fue positivo, pues quedaron fuera de la institución poetas de talento cuyo estilo de vida no congeniaba con el estilo que sus fundadores quisieron imprimir a la Arcadia. Al menos, Cristina Dudley y Teresa Zani, entre otras, vieron su labor poética reconocida en diversas antologías.

Abstract: The Accademia dell'Arcadia was, since its creation, an excellent omen for women who wished to practice writing. The Arcadia writers formed a large group that, although within the imposed canon, stood out for the quality of some of its components to the point of becoming part of the male anthologies not only at that time, but also in later centuries.

However, not everything was positive, since talented poets whose lifestyle did not match the style that their founders wanted to impose at the Arcadia were left out of the institution. At least Cristina Dudley and Teresa Zani, among others, saw their poetic work recognized in various anthologies.

\section{Introducción}

Angelo Gianni (1997) enumera tres posibles causas por las que las mujeres no participaron en el nuevo gusto barroco: según el autor, tal vez los excesos lingüísticos del barroco fueron demasiado para la modestia de las literatas del siglo, que a lo máximo que llegaron fue a participar, no sin esfuerzos, en las academias. Otra razón podría ser 
que las rimas de corte marinista no aparecieron en las antologías poéticas posteriores debido al rechazo que por éstas sintieron los literatos de los siglos venideros. Por último, siempre según el mismo autor, es que sí que existen rimas de este tipo hechas por mujeres, pero aún no han sido encontradas o estudiadas, hipótesis que tiene mucho sentido si observamos que la recuperación de autoras de otros siglos es un fenómeno relativamente reciente.

Sea como fuere, el uso y abuso de estas formas dio lugar a que el 5 de octubre 1690 se fundara en Roma la Accademia dell'Arcadia, en la que participaron escritores como Giovanni Mario Crescimbeni, Vincenzo Leonio, Gian Vincenzo Gravina y Giambattista Felice Zappi. Surgió como reacción antibarroca y recogió la herencia de las academias del Seicento (como la Academia Real, en 1674, fundada por la exreina de Suecia María Cristina). Su creación respondía también a un intento de "reforma" de la cultura italiana, que por aquel periodo se encontraba rezagada con respecto a la europea, y pronto se unieron los más importantes escritores italianos. Entre la enorme cantidad de academias que aparecían y desaparecían continuamente, la Arcadia consiguió imponerse en el Settecento como imagen de moderada racionalidad, modo de participación en la sociedad literaria y expresión de un buen gusto a menudo formal.

En la Arcadia se buscaba un estilo racional y clásico, aunque se conservaron algunos aspectos barrocos, visibles en la amplificación heroica y en el esfuerzo ingenioso de las canciones de Alessandro Guidi (1650-1712), durante mucho tiempo referencia de este nuevo gusto. Se rechazaron las soluciones más temerarias del Barroco, criticadas duramente por el jesuita francés Dominique Bouhours
(1628-1702) en Manière de bien penser dans les ouvrages de l'espirit (1687), pero aún se intentaba buscar el asombro.

La actividad de esta academia se desarrollaba en el bucólico estilo de vida de la antigua Arcadia, asumiendo cada socio un nombre griego ficticio. Como nota curiosa, ni la Arcadia se libró de las contradicciones del siglo, ya que tenía como emblema la siringa de Pan (mundo griego y pagano), y como protector al niño Jesús.

El ideal patronato que los primeros arcádicos reconocieron a la reina Cristina de Suecia suponía un excelente augurio para la posterior admisión de mujeres en la academia. No obstante, se sabe que no hubo mujer alguna en la fundación de la Arcadia, el 5 de octubre de 1690; tampoco se hizo referencia alguna a la admisión de mujeres cuando en 1695 se redactaron las leyes de la academia ni en el docto discurso Pro legibus Arcadum de Gravina, también en el mismo año.

Las primeras mujeres de las que se tiene constancia que entraran en la academia fueron la princesa napolitana Anna Beatrice Spinelli Carafa (Amaranta Eleusina) y Maria Selvaggia Borghini (Filotima Innia), ambas en $1691^{1}$. Borghini encarnaba el papel de la virgen erudita que ejercía la poesía por mero entretenimiento y se codeaba con literatos, como demuestra el hecho de que fuera alumna de Marchetti (Alterio Eleo), amiga de Redi (Anicio Traustio) y de Malagotti (Lindoro Elateo). La entrada de estas dos mujeres no supondría un cambio en la política de admisiones de la Arcadia, quedando como un hecho aislado y con mero carácter honorífico, debido a la excepcional posición de ambas mujeres. De hecho, no se con-

1. La admisión fue honorífica, pues eran grandes representantes de la aristocracia. 
serva documento alguno que certifique su participación en las actividades de la academia. En los años venideros serían muchas las admisiones honoríficas que responderían a criterios de representación o deferencia con grandes y poderosas familias: Anna Giuditta Febei (1691 Erminda Alicea), Rosa Agnese Bruni (1695 Galatea Beliminia), Virginia Rossi Alberici (1692 Acasta Cromonia), Passerini di Spello (Silvia Licoatide), Faustina degli Azzi (1691 Selvaggia Eurinomia), Aurora Sanseverino Gaetani (1691 Lucinda Coritesia) Giovanna Caracciolo di Santobuono (1696 Nosside Ecalia), Maria Settimia Tolomei Marescotti (1696 Dorinda Parasside).

Ninguna de las citadas anteriormente era residente en Roma ni asistía a las reuniones arcádicas. Únicamente dos mujeres formaron parte activa de estas reuniones: Maria Antonia Scalera Stellini di Acquaviva (1694 Aricia Gnateatide), protegida del duque Agostino Chigi, y Maria Casimira de Polonia (1699 Amiriscia Telea), que celebraba las reuniones arcádicas una vez al año en su palacio, tal como hiciera la propia Cristina de Suecia.

Habría que esperar hasta el periodo de la Arcadia gestionado por Crescimbeni para que se produjeran notables cambios. Crescimbeni, en su actividad como erudito y filólogo, había señalado en más de una ocasión la importancia de la producción femenina dentro de la tradición de la literatura italiana, si bien veremos más adelante que siempre dentro de unos límites. En su Istoria della volgar poesia, (publicada en seis libros en 1697) y después en los Comentari intorno alla storia della volgar poesia (cinco volúmenes entre 1702 y 1711), organizó una historia literaria italiana, a través de la lírica y, sobre todo, a través del petrarquismo como hilo conductor, que incluyó también a las poetas italianas. Se trata de un repertorio de diferentes textos poéticos desde los orígenes hasta el Seicento.

El segundo volumen recogió una serie de notas críticas sobre cien poetas antiguos y cincuenta todavía vivos que Crescimbeni aunó bajo el título Elogi. El tono encomiástico perfila, además, una serie de noticias biográficas sobre los autores y autoras tratados que se incorporarían en la edición de 1714.

La novedad que supone el libro de Crescimbeni es precisamente la de elaborar una especie de antología de la crítica anterior a él, agrupando los juicios que habían dado otros comentaristas. Para ello, se propuso reunir en un volumen a cien artistas vivos y cuarenta muertos que habían marcado el devenir de los estilos practicados hasta entonces (Crescimbeni, 1714: 3). En este sentido, Crescimbeni recopila muchas composiciones de poetas de diferentes épocas como ejemplos métrico-estilísticos de sonetos, estancias, canciones etc., al mismo tiempo que proporciona noticias biográficas sobre sus autores, colocándolas como piezas claves en la evolución poética de diferentes composiciones. Mas allá de su planteamiento histórico o su valor erudito, nos interesa el hecho de que Crescimbeni trace la tradición literaria italiana como continuidad de la literatura y de la lengua y que ese diseño no excluya a las poetas, sino que, al contrario, las convierta en ejemplos de esa tradición literaria y lingüística.

El trabajo de arqueología filológica que rescataba a las poetas del pasado constituía un argumento más en la polémica de la "renovación del gusto" y la revalorización de la cultura nacional en contra de la literatura francesa, que era leída sobre todo por mujeres, según apunta Crescimbeni: 
Y estas cosas especialmente las decimos por las damas, entre las cuales ha corrido hasta ahora más que en otra especie la alabanza, exhortándolas a todas a hacer a nuestra lengua la debida justicia que le hacen, además de las muchas damas en nuestra historia, muchas otras que podrían nombrarse; y en especial en Roma la marquesa Camilla Caprara Bentivogli, la marquesa Cleria Cavalieri Sacchetti y la condesa Flavia Teodoli Bolognetti ${ }^{2}$ (Crescimbeni, 1702: 300).

El hecho de que el "custode" de la Arcadia no tuviera ningún prejuicio contra las mujeres se hace patente cuando les concede entrada en la Accademia della Arcadia en 1700, diez años después de su fundación. Las mujeres que querían entrar a formar parte de la Academia, además de tener más de veinticuatro años, debían ser expertas en versificación y llevar una conducta de vida ejemplar.

El documento que certificaba la participación de las mujeres llegaría en 1708 de manos del mismo Crescimbeni, que ya había escrito en 1700 La bellezza della volgar poesia spiegata in Otto dialoghi. El texto, titulado la Arcadia, tenía dos grandes diferencias respecto al del 1700: la primera es que, mientras que en la primera versión únicamente había una sola mujer, en el segundo las protagonistas eran precisamente las mujeres; la segunda y más importante es que en la Arcadia las mujeres no sólo proponen cuestiones, sino que participan activamente en la vida de la academia, algo que no sucedía en La Bellezza della volgar poesia spiegata in Otto dialoghi, donde la mujer se limitaba a oír y recibir pasivamente los discursos.

La academia de la Arcadia continuó su existencia hasta finales del siglo XVIII y

2. Las traducciones, tanto de las citas como de las poesías posteriores, son mías. durante este tiempo fueron muchas las adhesiones de mujeres: Prudenza Gabrielli, Matilde Bentivoglio, Antonia Colleoni Vertova, Aurora Sanseverino, Caterina degli Obizzi, Caterina Rusca, Virginia Bazzani, Elena Balletti, Maria Elisabetta Strozzi Odaldi, Luisa Bergalli, etc. Esta lista se podría ampliar hasta las más de 450 firmas femeninas que participaron en la Arcadia desde la fundación hasta el siglo XVIII.

\section{Ilustres admitidas}

En Roma, lugar de nacimiento de la academia, la primera mujer que formó parte de la academia fue la condesa Prudenza Gabrielli Capizucchi (Elettra Citeria) en 1695. El motivo de su entrada se debió a la tradición que permitía a la cuñada o sobrina de un alto cargo eclesiástico formar parte de la vida social. Capizucchi no tuvo ningún problema para frecuentar la Arcadia, puesto que era sobrina del cardinal Giulio Gabrielli y cuñada del cardenal Galeazzo Mariscotti, éste último bien relacionado con Giovanni Battista Felice Zappi y Vincenzi Leonio da Spoleto, ambos fundadores de la academia.

Si bien su inicio en la escritura fue tardío si lo comparamos con otras arcádicas (contaba cuarenta años), su producción abarcó una buena cantidad de composiciones divididas entre sonetos, madrigales, canciones, etc. Los temas que trató no se alejaron de lo mencionado anteriormente y encontramos poesías donde expresó el dolor por la muerte del marido y la madre. Crivelli (2007) ha estudiado cómo las mujeres afrontaron la angustia y el luto y apunta que, en el caso de Capizucchi, resulta claro que el tono es mucho más cercano y familiar cuando los 
versos están dirigidos a su madre que los que dedica al marido, donde observa un tono más formal "siguiendo los cánones del elogio" (Crivelli, 2007: 114). He aquí el mencionado poema en el que expresa el duelo por la desaparición de la figura materna:

Crudo pensiero, intorno al duol mortale, Che l'alma ingombra, omai che più t'aggiri? Togliti dalla fredda urna fatale, Urna, che tutti chiude i miei sospiri.

Colei, donde trass'io la spoglia frale, Mercè di lui, che regge i sommi giri, Siede già nel suo seggio alto, immortale, Cinta il crin di piropi, e di zaffiri.

Vedila pur, come ne' giri eterni, In mezzo alle virtù, che le fur scorta, Lieta nel divin Sol tutta s'interni.

Or tu, il cener lasciando, a lei ti porta, Che pur dal Cielo a me con moti interni Parla, e qual già solea, m'ama, e conforta ${ }^{3}$ (Rime degli Arcadi, III, 1716: 108).

En cuanto a Petronilla Paolini Massimi, se encuentra entre las figuras más relevantes de este primer periodo arcádico. Nacida en 1663, fue hija de Francesco Paolini, barón de Ortonia dei Marsi, y de Silvia Argoli, de cuya familia salió también el matemático Andrea Argoli y el poeta Giovanni Argoli. Como sucede en otros muchos casos, las circunstancias que le tocó vivir en su vida marcaron su forma de hacer

3. Crudo pensamiento, en torno al duelo mortal / que el alma apesadumbra, ¿por qué deambulas? / Apártate de la fría urna fatal / Urna que encierra todos mis suspiros. / Esa, de donde saco los frágiles despojos, / A merced de él, que rige los altos círculos / Sentada en su alta sede, inmortal, / adornados los cabellos de piropos, y de zafiros. / Mírala cómo en los círculos eternos, / entre las virtudes, que la acompañaron, / Feliz en el divino Sol se adentra. / Ahora tú, las cenizas dejando, a ella te lleva, / Que desde el Cielo a mí con movimiento interior / Habla, y como solía, me ama, y conforta. poesía. Toda su producción se encuentra plagada de referencias biográficas y la belleza de sus versos denota una profunda tristeza. Su infancia fue especialmente dura, debiendo afrontar con cuatro años el asesinato del padre, aunque lo más duro estaría por llegar: poco después, con tan solo diez años, fue obligada por Clemente $X$ a contraer matrimonio con su tío, el marqués Francesco Massimi.

Comenzaría para Petronilla una época de infinita tristeza. Su tío, treinta años mayor que ella, era un hombre autoritario: no le permitía salir del Castel Sant'Angelo, una época que la poeta recordaría después como el "chiuso orrore"; poco después la encerraría en un convento del que no se le permitiría salir ni para asistir al entierro de uno de sus hijos.

A pesar de todo ello, y gracias a la ayuda de una "alta reina", que Morandini (2001) identifica con la figura de Cristina de Suecia, Petronilla resurgiría de las cenizas de su triste vida afirmándose mediante la sensibilidad de su poesía. Escribió textos para ocasiones religiosas, ceremonias oficiales, composiciones que podemos llamar ocasionales y dos dramas para música. Pero donde realmente destacó fue en la composición de sonetos y canciones bajo el prisma del petrarquismo. En ellos, la poeta encontró el esquema perfecto para dar rienda suelta a toda la amargura y el sufrimiento vivido con una profundidad capaz de conmover al crítico Walter Binni, quien en Sviluppo della poetica arcadica del primo settecento (1963) dijo de ella que fue una mujer que puso voz al sufrimiento de miles de romanas. En 1698 fue admitida en la Academia de la Arcadia con el nombre de Fidalma Partenide.

Entre sus composiciones más conocidas destaca por su temática el soneto Sdeg- 
na Clorinda ai femminili uffizi. Petronilla expresó en estos versos la suerte que corrían las mujeres, relegadas a labores consideradas "femeninas" por los hombres. Se deja entrever también cierto inconformismo y espíritu de lucha que aparece representado en el significativo nombre de Clorinda, de evocación tassiana:

Sdegna Clorinda a i femminili uffici chinar la destra, e sotto l'elmo accoglie i biondi crini e con guerriere voglie fa del proprio valor pompa a i nemici.

Così gli alti natali e i lieti auspici e gli aurei tetti e le regali spoglie nulla curando, Amalasonta coglie de' fecondi Licei lauri felici.

Mente capace d'ogni nobil cura ha il nostro sesso: or qual potente inganno dall'imprese d'onor l'alme ne fura?

So ben che i fati a noi guerra non fanno, né i suoi doni contende a noi natura: sol del nostro valor l'uomo è tiranno ${ }^{4}$ (Bergalli, 1725, II: 262)

Es por composiciones de este tipo que Cervone (1991) se atreve a decir, aunque siempre con cautela, que Petronilla Paolini fue un rostro transgresivo de la Arcadia femenina. Graziosi (1992), refiriéndose igualmente a este soneto, se aventura a hablar de tenue feminismo.

4. Desprecia Clorinda a los oficios femeninos / agachar la cabeza, y bajo el yelmo recoge / el rubio cabello y con guerreros deseos / hace del propio valor pompa a los enemigos. / Así la alta cuna y los felices auspicios / y los áureos techos y los reales restos / sin prestar atención, Amalasunta coge / de los fecundos liceos laureles felices. / Mente capaz de toda noble atención / tiene nuestro sexo: ¿qué potente engaño / de las empresas de honor las almas roba? / Sé bien que los hados a nosotras no nos desean guerra, / ni sus dones disputa a nosotros la naturaleza: / solo de nuestro valor el hombre es tirano.
Por último, destacamos entre quienes formaron parte de la Arcadia a Faustina Maratti Zappi que se encuentra, junto a Petronilla Paolini, entre las poetas más recordadas. Fue en vida célebre por el frustrado intento de secuestro por parte del segundogénito de la familia Sforza, Gian Giorgio Sforza Cesarini, que años más tarde regresaría a su vida reclamando la paternidad de uno de sus hijos, hecho que Faustina, ya casada desde hacía tiempo con el abogado y también poeta Giambattista Felice Zappi, siempre negó.

Las poesías recogidas en la obra de Recanati se atienen al gusto de la Arcadia y solo posteriormente se han recopilado otras de distinta temática. Proponemos aquí uno de sus sonetos más conseguidos, donde poesía y elemento biográfico se unen para cantar la prematura muerte del hijo:

Dov' è, dolce mio caro amato figlio; Il lieto sguardo, e la fronte serena?

Ove la bocca di bei vezzi piena,

E l'inarcar del grazìoso ciglio?

Ahimè! tu manchi sotto il fier periglio

Di crudel morbo, che di vena in vena

Ti scorre, e il puro sangue n' avvelena,

E già minaccia all' alma il lungo esiglio.

Ah! ch' io ben veggio, io veggio il tuo vicino Ultimo danno, e contro il ciel mi lagno,

Figlio, del mio, del tuo crudel destino!

E il duol tal del mio pianto al cor fa stagno, Che spesso al tuo bel volto io $\mathrm{m}^{\prime}$ avvicino, E nè pur d' una lagrima lo bagno ${ }^{5}$ (De Blasi, 1930: 387).

5. ¿Dónde está, amado y dulce hijo mío / la feliz mirada y la frente serena? / ¿Dónde la boca colmada de ternura / y el arco de la graciosa pestaña? / ¡Ay de mí! Tú sucumbes al fiero peligro / De la cruel enfermedad que por las venas te corre y la sangre pura te envenena, / Y ya amenaza el alma el largo exilio. / ¡Ah! ¡Yo bien veo, yo veo 
Tratar a todas las autoras que se dieron cita en la Arcadia requeriría un extenso trabajo, pues fueron más de 450 las mujeres que contribuyeron con sus escritos a engrandecer el nombre de una de las academias más longevas. No obstante, en un trabajo que pretende demostrar la existencia de un número de escritoras mayor del que se recoge en las historias de la literatura, es menester mencionar al menos los nombres (con sus correspondientes apelativos arcádicos) de aquéllas que pertenecieron al arco temporal que aquí se trata, mostrando así el arduo trabajo por realizar para desenterrar a estas mujeres olvidadas: Anna Giuditta Febej da Orvieto (Erminda Alicea) Vivente; Aurora Sanseverino Caetani (Lucinda Caritesia); Antonia Vertova CoIleoni (Elcinda Efireana); Cristina Regina di Svezia (Basilissa); Elena Balletti (Mirtinda Parasside); Elisabetta Credi Fortini (Alinda Panichia); Elisabetta Gerolami Ambra (Idalba Corinnetta); Emilia Ballati Orlandini (Eurinda Anodimia); Faustina degl'Azzi ne'Forti (Selvaggia Eurinomia); Fidalma María Vagnucci da Cortona (Eurilla Arcnesa); Gaetana Passarini (Silvia Licoatide); Giovanna Caracciolo (Nosside Eccalia); Giulia Lama (Lisalba); Giulia Serega Pellegrini (Erminia Meladia); Ippolita Cantelmi Carrafa (Elpina Aronte); Luisa Bergalli (Irminda); María Ardoini Lodovisia (Getilde Faresia); María Buonaccorsi Alessandri (Leucride Sonide); María Selvaggia Borghini (Filotima Innia); María Settimia Tolomei Marescotti (Dorinda Parasside).

tu último dolor cercano / Y al cielo me lamento, hijo, de nuestro cruel destino! / Y es tal el dolor de mi llanto que al corazón estanca, / Que a menudo a tu bello rostro me acerco / Y ni siquiera con una lágrima lo humedezco.

\section{Ilustres excluidas}

La lista anteriormente mencionada pudo haber sido mayor si alguna de las poetas que intentó entrar sin éxito en dicha institución lo hubiera conseguido. No obstante, llama la atención que en la misma antología de Recanati de 1716 se dieran cita varias autoras más o menos desconocidas cuya solicitud fue desestimada Si bien nos centraremos en Cristina de Northumberland Paleotti y Teresa Zani, ambas oriundas de Bolonia, hubo muchas otras que permanecieron al margen de la gran academia romana: Elena Maria Cavassi Tracanelli, Verginia Catelani, Orsola Maria Troni Poggiolini, etc.

Volviendo a Paleotti y Zani, ninguna de las dos aparecía en Notizie degli scrittori bolognesi (1714) del padre Pellegrino Orlandi, quien no encontró mujeres de talento a las que situar junto a las escritoras del Duecento y Trecento. Hemos de tener en cuenta que para hablar de estas dos mujeres hemos de considerar la situación en Bolonia, donde para las escritoras era aún más difícil formar parte de la academia. Mientras que en la Arcadia romana ya se admitían mujeres, en la Colonia Renia únicamente eran admitidas aristócratas y sólo como espectadoras en la fiesta de inauguración. La primera mujer que consiguió formar parte de la academia boloñesa fue Laura Bassí.

6.Laura Maria Catharina Bassi (1711-1778) fue una científica boloñesa conocida principalmente por su exitosa trayectoria en el campo de la física. Ha pasado a la historia por ser la primera mujer en ocupar un puesto de profesora de física en la prestigiosa Universidad de Bolonia, que le concedió un doctorado honorario, así como por haber sido miembro de la Academia de Ciencias de Bolonia. También compuso poemas y trató la filosofía. 
Cristina Dudley despertó en su momento más interés por su persona que por su obra, algo que solo en época relativamente reciente se ha visto compensado. Hija de Carl Dudley, duque de Northumberland, ciertamente su vida no fue lo que se esperaba de alguien de su posición: se le cuentan infidelidades (incluso con una hija fuera del matrimonio), frecuentes salidas de tono que no respetaban protocolo previsto, así como otras aventuras que le granjearon una fama que, muy probablemente, fue decisiva para desestimar su entrada en la Arcadia.

No obstante, y dejando a un lado el juicio de críticos como Corrado Ricci, más interesado en la moral que en la literatura, Cristina fue poeta de reconocido talento y sus composiciones aparecieron en la mencionada antología de Recanati, las Rime scelte di poeti illustri de' nostri tempi, de Bartolomeo Lippi y las antologías de Bergalli (1726) y Fachini (1824), entre otras. En años recientes ha recibido atención por parte de Graziosi (2008) y Conte (2008) con excelentes resultados.

Es precisamente gracias a estos últimos estudios que podemos apreciar la escasa obra de Cristina desde un punto de vista distinto al que ha sido tradicionalmente propuesto. En la obra de Recanati, que marcó el devenir en su tratamiento, las poesías aparecían colocadas como si de un cancionero se tratara, con los sonetos de juventud primero y los de la vejez en último, dando a entender un supuesto arrepentimiento por su díscola vida. He aquí uno de los más conocidos, donde expresa el sufrimiento que le produjo la ruptura con el príncipe Lorenzo Onofrio Colonna (aunque nunca se menciona expresamente su nombre), con quien tuvo una hija:
Armato di rigor l'augusto ciglio a me sempre crudel, Prencipe amato, sordo a' miei preghi e al mio desire ingrato, mai non torrete da pietà consiglio?

Soffrirò pur il doloroso esiglio, sosterrò pur vostra fierezza, e irato mai non fia questo cor, benchè ostinato ad adorare in voi il suo periglio.

Pria che mutar pensiero io morir voglio. Voi foste infido: io, lassa, troppo amante: sia il pentimento uguale al nostro orgoglio.

Ed ambi al do d'amor prostati avante quali cose di voi narrar non gli voglio! Voi non di me, ch'io fui fida e costante ${ }^{7}$ (Recanati, 1716: 19).

La otra poeta "aventurera", como la llama Graziosi (1998), Teresa Zani, nació en 1685 en el seno de una acaudalada familia, no en vano su padre, Paolo Zani, era uno de los abogados más conocidos de su Bolonia natal. Se decidió su matrimonio con el conde Agesilao Mariscotti con tan solo trece años ${ }^{8}$, al mismo tiempo que su viuda madre contraía segundas nupcias con su suegro Vincenzo. Los matrimonios convenían a ambas partes, ya que los Zani conseguían entrar en los selectos círculos aristocráticos gracias al título de condesa,

7. Armada de rigor la augusta mirada, / siempre cruel conmigo, Príncipe amado, / sordo a mis suplicas e ingrato a mi deseo, / ¿acaso no obtendréis consejo de la piedad? / Sufriré incluso el doloroso exilio, / soportaré también vuestra soberbia, y airado / nunca estará mi corazón, aunque se obstine / en adorar en vos su peligro. / Antes que cambiar de idea prefiero morir. / Vos fuisteis desleal, yo, infeliz, os amé demasiado: / sea el arrepentimiento igual a nuestro orgullo. / $\mathrm{Y}$ ambos al dolor de amor nos postraremos después, / ¡cuántas cosas no quiero narrar de vos! / Vos no podréis de mí, que yo fui fiel y constante.

8. Canonici Fachini difiere en este dato. Según ella, Faustina habría contraído dicho matrimonio en segundas nupcias, tras haber resultado fallido el primero con Bettini. 
y los Mariscotti podían lavar su imagen de libertinos, a la vez que llenaban sus arcas con el dinero de la rica familia. El revuelo que levantaron en Bolonia estos matrimonios fue notable, aunque los escándalos no terminaron ahí: en 1708 murió el conde Mariscotti y, cuatro años después, Teresa, como ya hiciera su madre, no se resignó a ser viuda y se casó de nuevo con otro conde, el modenés Carlo Felice Scapinelli. En las poesías de Zani encontramos reflejado el complicado tema de los matrimonios de conveniencia que ella misma sufrió, de hecho, en uno de sus sonetos, llega a afirmar que si un hombre le gustaba se lo compraría con oro, lo que puede ser interpretado como una referencia a los motivos de su propio matrimonio.

A pesar de que en una mujer tratar dicho argumento suponía una novedad, Zani no hizo más que exportar al género lírico un tema que ya triunfaba, a la vez que levantaba ampollas, en comedias y melodramas. El matrimonio entre personas de distinta clase social había sido llevado a escena entre 1681 y 1698 por Antonio María Monti en Amor torna un s' al so y Diporti d'amore in villa y por Lelio María Landi en Inganni amorosi scoperti in viIla. Dice Graziosi (1994) que al pasar del teatro a la poesía se perdieron las connotaciones cómicas, a la vez que se fijaron las características de una nueva figura pastoral-femenina: rica, amorosa, poeta, volitiva y no conformista.

Zani rompió en sus poesías con el modelo petrarquista y esto le costó no ser admitida en la academia de la Arcadia. El modelo de mujer que la Arcadia crescimbeniana aceptaba era el que se hallaba en consonancia con el espíritu moralista del nuevo guardián, que no estaba dispuesto a aceptar que se tratara el matrimonio como un contrato comercial. La poesía debía seguir el modelo de Petrarca y lo máximo que se concedía era la evocación heroica de funestas gestas familiares, como fue el caso de Petronilla Paolini. Todo argumento que escapara del férreo código era automáticamente eliminado y olvidado. Es por ello que temas como el divorcio no tenían cabida, mientras que el canto a la muerte del marido era algo recurrente.

El problema derivado de esta exclusión fue que también quedó fuera del amplio círculo de influencia de la gran academia, lo cual repercutió en la fortuna editorial de su trabajo. No se la menciona en las Rime degli Arcadi, ni en las Vite ni en las Notizie degli Arcadi morti; tampoco se la incluyó en las diversas colecciones de poesías de ocasión, ni en la red epistolar, así como tampoco fue invitada a los salones literarios que la Arcadia organizó por toda Italia.

La poca fortuna en materia de publicaciones quedó definitivamente contrastada cuando el arcádico Pier Jacopo Martello (Mirtilo Dianidio) se vanaglorió de haber convertido a Zani en poeta de un año a otro. La acusación ponía en duda la autoría de los textos, algo para nada nuevo, ya que antes la habían sufrido otras poetas incluidas en la antología de Recanati. Calumnias similares se han producido a lo largo de la historia: la mujer que conseguía publicar era siempre acusada de tener un amante, o incluso a alguien que escribía por ella, como le sucedió a la arcádica Aurora Sanseverino, cuyos sonetos se decía que habían sido escritos por el abad Nicola Giuvio (Eupidio Siriano). Lo más grave fue que, al final, los versos de Zani aparecieron en la antología de Martello como si de verdad le hubieran sido dirigidos a ella.

Las primeras poesías de Teresa Zani las encontramos en las dos antologías feme- 
ninas del XVIII, Poesie italiane di rimatrici viventi de Recanati (1716), donde se recogen algunos sonetos y canciones, y en Componimenti poetici de Bergalli. En 1739 Agostino Gobbi incluyó algunas composiciones en Scelta di sonetti e canzoni y ya en el XIX en Sonetti di ogni secolo della nostra letteratura (1834), de Francesco Ambrosoli, y en Storia del sonetto italiano de 1839.

Al igual que sucede con las composiciones de Cristina Dudley, en la antología de Recanati los sonetos de Zani estaban organizados como un canzoniere. Se perfilaba en ellos la imagen de una joven enamoradiza y deseosa de casarse por amor, sin importarle que la clase social del amado no fuera tan ilustre como la suya. Entre los más conocidos se encuentra el primer soneto que aquí se reproduce, en el cual se autorretrata siguiendo el nuevo gusto por este tipo de composiciones que había introducido Maggi en Bolonia, y que tan buen resultado daría en la pluma de Alfieri, Foscolo o Manzoni:

Di quattro lustri e, come son, disciolta dai genitori miei, che terra or sono, posso, a mia voglia, o saggia siasi o stolta, o pietade impetrare o almen perdono.

Piacemi la mia rete, a ch'io son còlta, garzon di viso ognor modesto e prono e chiamo il ciel, che i giuramenti ascolta, che s'ei sposa m'accetta, a lui mi dono.

Che l'invidia dirà? Famosi e chiari avi ei non vanta al par di me, ma nacque tal che dovria di me vantarli al pari;

E poi sacro ha l'ingegno, e poi dell'acque bee d'Elicona, e poi d'onesti e rari atti adorno m'apparve; e poi mi piacque ${ }^{9}$ (Recanati, 1716: 227).

9. De cuatro lustros y, como soy, libre / de mis padres, que tierra ahora son, / puedo, a mi antojo, sea sabia o necia, / o suplicar piedad o al menos

\section{Conclusión}

Fueron muchos los críticos que opinaron que la Arcadia supuso el despertar de la poesía tras los excesos en los que había caído un barroco "saturado". No pensó lo mismo el crítico italiano Benedetto Croce (1946), que rechazó tanto la poesía barroca como su reacción arcádica, afirmando que en un siglo preocupado por la ciencia y la "mortificación espiritual", no quedó lugar para la poesía. Croce, siguiendo la línea marcada por Leopardi, que dijo que después de Tasso no hubo ningún gran poeta, sino que tan sólo se escribieron versos sin poesía, afirmó que la Arcadia no se podía considerar el despertar de la poesía del que hablaban algunos críticos, ya que nació como reacción y la poesía no es nunca reacción, sino producción positiva.

A pesar de que se antoja un débil argumento como para despreciar toda la poesía arcádica, es cierto que la más famosa academia cometió algunos "errores", si es que así se pueden llamar, que le impidieron alcanzar una dimensión mayor. Uno de los reproches que se pueden dirigir hacia la institución romana es que, poco a poco, se vio invadida por una excesiva mundanidad. Cuando las relaciones sociales tomaron un papel más que relevante dentro de la institución, la Arcadia perdió en gran medida el objetivo de "mundo idílico y poético" al que aspiraba en un principio. Como sostiene Graziosi:

perdón. / Me gusta la red de la que soy presa, / joven de rostro siempre modesto y servil / y clamo al cielo, que los juramentos escucha, / que si me toma por esposa, a él me entrego. / ¿Qué dirá la envidia? De famosos e ilustres / antepasados él no puede jactarse, pero nació tal / que debería jactarse por igual. / Y sacro ingenio posee, y de las aguas / bebe de Helicón, y con honesto y singular / comportamiento se me muestra; y me gustó. 
Virginidad y reclusión fueron en cambio excepciones en la más mundana sociabilidad de la Arcadia, e incluso la poesía de los compañeros pastores acompañó con maliciosa indulgencia las etapas de una regulada inserción mundana: matrimonio, actuaciones y representaciones, embarazos y partos (Graziosi, 1992: 325).

Centrándonos en el caso de la participación femenina, la crítica más destacable que se puede lanzar es la poca variedad en las temáticas de las composiciones femeninas. Si bien es cierto que hubo poetas de cierto relieve, ninguna se salió del conocido canon petrarquista. No hubo entre ellas -y eso fue tal vez lo que faltóuna poeta que se saliera de este canon, ni siquiera que lo invirtiese, como ya hizo Gaspara Stampa. La explicación a este excesivo petrarquismo no es fácil y puede responder a varios motivos: el primero de ellos es que el petrarquismo estuvo presente desde que se empezó a gestar la Arcadia, en el tiempo de Cristina de Suecia, gracias a la enorme admiración que la reina e inspiradora de la academia sentía por el poeta florentino.

El segundo es la puesta en circulación de textos escritos por las grandes poetas de siglos anteriores, que respondían al canon derivado de la poesía de Petrarca. Especialmente importante fue la obra de Bulifon, que publicó en Nápoles entre 1692 y 1692 las Rime de las mejores poetas del Cinquecento. Entre ellas se encontraban los grandes nombres de la poesía lírica: Laura Terracina, Isabella Andreini, Tullia d'Aragona, Isabella Morra, Laura Battiferri, Veronica Gambara y Vittoria Colonna.

La publicación de ésta y otras antologías femeninas pusieron de nuevo en circulación un código lingüístico y una temática hipercodificada fácil de usar. El repetido uso de esta fórmula hizo que la Arcadia adoleciera de cierta carencia de originalidad en los textos.

A pesar de éstas y otras críticas que se pueden reprochar a la institución, no cabe duda de que la academia de la Arcadia supuso para la mujer un espacio donde pudo satisfacer las necesidades literarias que le fueron negadas en el resto de las instituciones culturales de la época.

\section{Bibliografía}

Bergalli, L., Componimenti poetici delle piu' illustri rimatrici d'ogni secolo, raccolti da Luisa Bergalli, Antonio Mora, Venezia 1716.

Binni, W., "Sviluppo della poetica arcadica nel primo Settecento", Rassegna della letteratura italiana, VII (LIX), 2, 1955, pp. 341-348.

Cacciari, C., Zanelli, G., Faustina Maratti tra Roma ed Imola: immagine pubblica e tormenti privati di una poetessa italiana del 700, en La mandragora, Imola, 1995

Canonici Fachini, Prospetto biografico delle donne italiane, Tip. Di Alvisopoli, Venezia 1824.

Cracolici, S., "Le donne illustri di Faustina Maratti Zappi", Giornale storico della letteratura italiana, CXCV (650), 2018, pp. 179-214.

Crescimbeni, G.M. (Ed.). Prose degli arcadi. Roma: Antonio de' Rossi, 1718. Crescimbeni, G.M. (Ed.), Rime degli Arcadi, X. Antonio de' Rossi, 1747.

Crivelli, Tatiana, "«Figli, vi lascio! E nel lasciarvi tremo». Sui domestici lutti poetici delle 'Pastorelle' d'Arcadia", Rassegna europea di letteratura italiana, vol. 20-30, 2007, pp. 109-124. 
Croce, B., La letteratura italiana del Settecento, Laterza, Bari 1949, pp. 59-75.

Croce, B., Nuovi Saggi Sulla Letteratura Italiana Del Seicento, Laterza, Bari, 1968, pp. 169-172.

De Blasi, J., Scrittrici italiane dalle origini al 1800, Nemi, Firenze 1830, pp. 384-391.

Fantuzzi, G., Notizie Degli Scrittori Bolognesi: 6, Stamperia di San Tommaso D'Aquino, Bologna ,1788, pp. 229-264.

Ferri, P. L., Biblioteca femminile italiana, Tipografia Crescini, Padova 1842.

Gianni, A., Anch'esse "quasi simili a Dio": le donne nella storia della letteratura italiana, in gran parte ignote o misconosciute dalle Origini alle fine dell'Ottocento, Baroni, Viareggio-Lucca 1997.

Gobbi, A., y Baseggio L., Scelta Di Sonetti E Canzoni De' Piu Eccellenti Rimatori D'ogni Secolo. Quarta Edizione Con Nuova Aggiunta [compilata Da Agostino Gobbi]: [parte Quarta] Rime D'alcuni Illustri Autori: Voir: Rime, Sous La Cote: Y 81768 Inv 3609-3610], L. Baseggio, Venezia 1739, pp. 169-198.

Graziosi, E., "Restauro d'autore: Teresa Zani contessa e rimatrice bolognesa", La Rassegna della letteratura italiana, XCVII, 1993, pp. 114-152.

Graziosi, E., Due poetesse avventuriere, La cultura fra Sei e Settecento. Primi risultati di una indagine, a cura di E. Sala Di Felice e L. Sannia Nowé, Modena, Mucchi, 1994, pp. 123-136.

Graziosi, E., Avventuriere a Bologna: Due Storie Esemplari, Mucchi, Modena, 1998.

Greco, G. R., "Una Dama Bolognese Del 17. Secolo: Cristina Dudley Di Northumberland Paleotti", I/ Carrobbio, XIX-XX, 1993-1994, pp. 185-202.
Gronda, G. (Ed.), Poesia italiana del Settecento, UTET, Torino 1989, pp. 152-154.

Maier, B. (Ed), Lirici del Settecento, Ricciardi, Milano-Napoli, 1959, pp. 100-101.

Maier, B., Faustina Maratti Zappi, donna e rimatrice d'Arcadia, L'Orlando, Roma, 1954.

Maier, B., Rimatori d'Arcadia: Giambattista Felice Zappi, Faustina Maratti Zappi, Eustachio Manfredi, Carlo Innocenzo Frugoni, Del Bianco, Udine, 1972.

Manfredi, E. (Ed). Rime d'alcuni illustri autori viventi, aggiunte alla Scelta d'Agostino Gobbi [di cui formano la quarta parte] e in questa terza edizione accresciute, Lorenzo Baseggio, Venezia, 1711.

Morandini, G., Sospiri e palpiti. Marietti, Genova, 1820.

Muscetta, C. y Massei, M. R. (Ed), Poesia del Settecento, vol. I, Einaudi, Torino 1967, pp. 914-919.

Recanati, G. B., Poesie italiane di rimatrici viventi raccolte da Teleste Cipatisiano pastore arcade, Venezia, 1716.

Ricci, C., Una Illustre Avventuriera: (Cristina Di Nortumbria), Treves, Milano, 1891. Ricci, C., Anime Dannate, Fratelli Traves, Milano, 1918, pp. 111-258.

Romano Cervone, A. T., "Faustina Maratti Zappi e Petronilla Paolini Massimi: I'universo debole della prima Arcadia romana", en Atti e memoria dell'Accademia degli Arcadi, 9 (2-4). Roma, 1991, pp. 169-176.

Tioli, G., Cronica di d. Giovanni Tioli, che comincia dall'anno 1642 per tutto li 23 Iuglio 1708 giorno della sua morte, MS, 17th/18th century, I-Bu, MS No. 3847. 